\title{
TEK KATMANLI GRAFEN TABAKALARIN EĞİLME VE TİTREŞIMİ
}

\author{
Çiğdem DEMİR*, Ömer CIVALEK
}

Akdeniz Üniversitesi, Mühendislik Fakültesi, İnşaat Mühendisliği Bölümü, Antalya, Türkiye

\begin{tabular}{ll}
\hline Anahtar Kelimeler & Özet \\
\hline Yerel olmayan & Elastik bir ortam ile temas halinde olan grafenler uygulamada sıklıkla \\
elastisite teorisi, & kullanılmaktadır. Bu çalışmada, yüksek mertebeden elastisite yöntemleri \\
Nano mekanik, & kullanılarak nano ya da mikro boyutlu grafen tabakaların frekansları ve \\
Grafen tabakalar, & deplasmanları hesaplanmıştır. Grafen tabakanın bulunduğ ortam Winkler- \\
Serbest titreşim analizi & Pasternak zemin ile modellenmiştir. Yerel olmayan elastisite teorisi boyut etkisini \\
& dikkate almak için kullanılmıştır. Çözümlerde hem Navier yöntemi hem de ayrık \\
& tekil konvolüsyon yöntemi kullanılmıştır. Boyut etkisinin diğer parametrelere bağlı \\
& olarak titreşim ve eğilme üzerindeki etkisi incelenmiştir. \\
\hline
\end{tabular}

\section{BENDING AND VIBRATION OF SINGLE-LAYERED GRAPHENE SHEETS}

\begin{tabular}{l}
\hline Keywords \\
\hline Nonlocal elasticity theory, \\
Nano mechanic, \\
Graphene sheets, \\
Free vibration analysis
\end{tabular}

\section{Giriş}

Son zamanlarda deneysel ve teorik olarak yapılan çalışmalarda nanoyapılara fazlaca yer verilmektedir. Bunun en önemli nedenlerinden biri bu küçük ölçekli yapıların mekaniksel, elektriksel, elektroniksel ve ısısal anlamda olağanüstü özelliklere sahip olduğunun tespit edilmesidir. $\mathrm{Bu}$ nedenle de nanoyapıların havacılık, biyomedikal, biyomekanik, biyoelektrik gibi çok geniş uygulama alanlarına sahip olacağ öngörülmektedir. Özellikle mikro ve nano ölçekli alanlarda teknolojinin hızlı gelişebilmesi ancak atomik kuvvetleri ve küçük ölçek etkisini göz önünde bulunduracak çözüm yöntemlerine bağlıdır. Bu etkilerin ihmal edilmesi yanlış çözümlere dolayısıyla yanlış tasarımlara neden olabilir. Moleküler dinamik gibi bazı yöntemler atomik uzunluk ve küçük boyut etkisini dikkate almaktadır(Sun ve Zhang, 2003; Zhu vd., 2007; Liang vd., 2007). Fakat bu çözüm yöntemleri çok büyük ve çok sayıda denklemler içermektedir bu da zaman kaybına neden olmaktadır. Bu nedenle sürekli ortam mekaniği daha fazla tercih edilmektedir. Bunlardan en iyi ve yaygın olarak bilinen ve küçük ölçek etkisini de dahil eden sürekli ortam modeli,
Eringen'in yerel olmayan elastisie teorisidir(Eringen ve Edelen, 1972; Eringen, 1983). Büyük denklem takımlarına gerek kalmadan nano boyutlu yapıların davranışlarını yaklaşık olarak tahmin edebilmektedir. Eringen'e göre bir noktadaki gerilme diğer bütün komşu noktaların şekil değiştirmelerinin bir fonksiyonudur. Atomlar arası kuvvetler ve atomik uzunluk doğrudan malzeme parametresi olarak bünye denklemlerine etki etmektedir. Bu nedenle yerel olmayan elastisite teorisinin günümüzde ve gelecekte önemli bir role sahip olduğu düşünülmektedir.

Karbon içeren birçok malzeme (karbon nanotüp, fulleren, nanoring) grafen tabakanın deforme hali olması nedeniyle, grafen tabakaları yapabilmek ve anlayabilmek deneysel çalışan araştırmacıların temel çalışması konusu olmuştur. Grafen tabakalar bize iki boyutlu karbon nano yapıları anlamak için yeni bir ufuk açmıştır. Grafen yeni nesil nanoelektronik cihazlar için yeni bir malzeme olarak düşünülebilir.

Tek katmanl grafen tabakalar birbirlerine kovalent bağ ile bağlanmış karbon atomlarından oluşan bal peteği görünümlü yapıdadır. Tek atom kalınlığına

\footnotetext{
* ilgili yazar/Corresponding author: cigdemdemir@akdeniz.edu.tr
} 
sahip olan grafenler, grafitin temel yapı taşıdır. Tek duvarlı ve çok duvarlı grafen tabakaların polimer kompozit gibi bir elastik zemine gömülü olduğunda ana malzemenin mukavemetinin arttırılması sağlanmiş olur.

Elastik ortam ile çevrelenme genellikle Winkler zemin olarak modellenmektedir. Winkler tipi modellemede zemin, yakın aralıklı birbirinden bağımsız, dikey doğrusal yay dizisi olarak tanımlanabilir. Zemin parametresi, yayların sertliği ile temsil edilmektedir (Avcar, 2010). Bununla birlikte Winkler modeli mekaniksel davranış için kaba bir yaklaşımdır. Yaylar arasındaki etkileşim dikkate alınmadığından zeminin bütünlügünü ve devamlılığını sağlayamamaktadır. Daha gerçekçi modelleme iki parametreli zemin modeli ile sağlanabilmektedir. Bu modellerden biri de Pasternak zemin modeli olarak anılmaktadır. Winkler zemin modeline ilave olarak yaylar arasına sıkıștırılamaz bir kesme tabakası eklenmiştir (Avcar, 2016a; Avcar, 2016b; Civalek ve Demir, 2009.).

Birçok araştırmacı farklı plak teorileri (klasik , birinci derece kayma deformasyon, üçüncü derece kayma deformasyon) ile nano ve mikro boyuttaki plaklar ile ilgili çalışma yapmıștır (Aksencer ve Aydoğdu, 2011; Alibeigloo, 2011; Ansari vd., 2011; Jomehzadeh vd., 2011; Malekzadeh vd., 2011a-b; Narendar, 2011; Pradhan ve Phadikar, 2009a; Tsiatas, 2009;). Klasik (Kirchoff) plak teorisinde kayma gerilmeleri ve șekil değiştirmeleri ihmal edilmektedir. Birinci derece kayma deformasyon (Mindlin) teorisine göre düşey normal gerilmeler ihmal edilirken, düşey kayma gerilmeleri plak kalınlığı üzerinde lineer olarak değişmektedir. Plağın alt ve üst sınırında kayma gerilmelerinin olmama şartı ise kayma düzeltme katsayıları ile sağlanmaktadır. Kayma düzeltme faktörü gerektirmeyen ve kayma gerilmelerinin parabolik olarak değişen plak teorisi ise üçüncü dereceden kayma deformasyon (Reddy) teorisidir (Ventsel ve Krauthammer, 2001; Wang vd., 2000).

\section{Bilimsel Yazın Taraması}

Son yıllarda teknolojide yaşanan hızlı gelişmeler, Son yıllarda teknolojide yaşanan hızlı gelişmeler, mikro ve nano boyutlu yapılar üzerindeki ilginin artmasına yol açmıştır. Bu yapıları en verimli biçimde kullanabilmek için tasarım aşamasında mekanik davranış karakteristiklerinin çok iyi bir biçimde bilinmesi gerekir. Bu tip yapıların analizinde iki temel yaklaşım, atomik seviyede oluşturulan modeller ve sürekli ortam mekaniği modellemesidir. Moleküler dinamik simülasyonu gibi atomik düzeyde kurulan modellerde, sadece belirli sayıda atomdan meydana gelmiş basit sistemler incelenebilmekte ve hesaplamalar çok uzun sürmektedir. Dolayısıyla, bu konu üzerinde çalışan araştırmacılar, modelleme aşamasında sürekli ortam mekaniği yaklaşımlarını kullanmayı tercih etmeye başlamışlardır. Aşağıda konuyla ilgili literatürde yer alan bazı çalışmalar sunulmuştur
Pradhan ve Murmu (2010) elastik zemine gömülü tek katmanlı grafen tabakaların burkulması yerel olmayan elastisite teorisine bağlı olarak yapılmıştır. Zemin tipi olarak Winkler ve Pasternak zemin tipi düşünülmüştür. Grafenin burkulma yükünde küçük ölçek katsayısının ve zemin parametresinin etkili olduğunu ayrıca zemin sadece Winkler olarak modellendiğinde zemin parametresinin artmasıyla yerel olmayan etkinin azalış ve artışlar gösterdiğini belirtmişlerdir.

Aghababaei ve Reddy (2009) plakların eğilme ve tireşim analizini yerel olmayan elastisite teorisi ile gerçekleştirmişlerdir. Plak modeli olarak üçüncü derece kayma deformasyon teorisi kullanılıștır. Sonuç olarak Kirchoff, Mindlin ve Reddy plak teorileri sayısal olarak karşılaştırılmıştır.

Pradhan ve Phadikar (2009b) tarafından çok katmanlı grafen plakaların serbest titreşim analizlerini yerel olmayan elastisite teorisine dayalı olarak gerçekleştirmişlerdir. Grafen plakaların polimer bir matris içerisinde olduğunu dikkate alarak bunları ortotropik ince plak gibi modellemişlerdir. Titreşimde, yerel olmayan etkinin yüksek modlarda daha etkin olduğunu, ayrıca polimer matrisin rijitliği arttıkça yerel olmayan etkilerin azaldığını ve kayma tabakasının etkisinin Winkler tabakasından daha fazla olduğunu belirtmişlerdir (Pradhan ve Kumar, 2010; Reddy, 2007; Ansari ve Rouhi, 2012; Ansari vd., 2010a; Ansari vd., 2010b; Ansari vd., 2011)

Ansari vd. (2010b), tek katmanlı grafen tabakaların serbest titreşimi ile ilgili çalışmışlardır. Moleküler dinamik sonuçlarla yapılan karşılaştırma sonucunda yerel olmayan parametrenin grafen tabakanın geometrik değişkenlerinden bağımsız olduğu sonucuna varılmıştır.

Samaei vd. (2011), tek katmanlı grafen plakaların burkulma analizini yerel olmayan elastisite ve Mindlin plak teorisini kullanarak gerçekleștirmişlerdir. Grafen plakaların elastik bir zemin üzerinde olduğunu Pasternak zemin modelini kullanarak dikkate almışlardır. $\mathrm{Bu}$ duruma ait yönetici denklemleri çıkartarak tüm kenarları basit mesnetli tek katmanlı grafen plaka için burkulma problemini analitik olarak çözmüşlerdir. Elde ettikleri sonuçları, grafiksel formda boyutsuz burkulma yükü oranının (yerel olmayan boyutsuz burkulma yükünün yerel (klasik) boyutsuz burkulma yüküne oranı) değişimi şeklinde sunmuşlardır. Grafen plakanın boyunun artmasıyla bütün burkulma modları için bu oranın arttığını ve mod sayısı arttıkça da bu oranın azaldığını göstermişlerdir. Ayrıca, hem Winkler hem de kayma parametresi değerlerindeki artışın bu oranda azalmaya neden olduğunu belirtmişlerdir. Burkulma analizi literatürde farklı parametre ve değișkenlerle çalışılmıştır (Babaei ve Shahidi, 2011; Farajpour vd., 2011a-b; Hashemi ve Samaei, 2011; Civalek ve Demir, 
2011; Civalek ve Demir, 2016; Mercan ve Civalek, 2016a)

Elastik zemin üzerindeki tek katmanlı grafen plakaların serbest titreşim analizi değiştirilmiş gerilme çifti teorisine dayalı olarak Akgöz ve Civalek (2012) tarafından gerçekleştirilmiştir. Yönetici denklemler ve sınır koşulları Hamilton prensibi yardımıyla elde edilmiştir. Elastik zemin ile grafen plaka arasındaki etkileşim Pasternak zemin modeli ile hesaba katılmıştır. Zemin parametresi ve ilave malzeme boyut ölçek parametresi değerlerinin artmasıyla doğal frekans değerlerinin de arttığını tespit etmişlerdir. Literatürde titreşim ve eğilme analizi üzerinde durulmuş pek çok çalışma bulunmaktadır (Civalek ve Akgöz, 2013; Akgöz ve Civalek, 2014; Liew ve Wang, 2007a-b; Liew vd., 2008; Liew vd., 1995; Lim ve Liew, 1995; Lim vd., 1996; Wang ve Wang, 2011; Wang ve Arash, 2012; Wang vd., 2011; Ma vd., 2008; Reddy ve Pang, 2008; Demir ve Civalek, 2015; Demir ve Civalek, 2016; Akbaş, 2016ab-c; Kocatürk ve Akbaș, 2013)

Grafen, karbon atomunun bal peteği görünümlü yapılarından bir tanesine verilen isimdir (Şekil 1.). karbon atomu periyodik tablodaki en ilginç elementlerden biridir (Salvetat vd., 1999). Özellikle karbon nanotüpler ve C60 (fulleren) molekülleri ilk sentezlendikleri yıllardan günümüze kadar katı hal fiziğini son derece aktif araştırma alanları arasına girmiştir (Şimşek, 2010; Williams ve Adams, 2007; Zhang vd., 2005, Zhang vd., 2007).

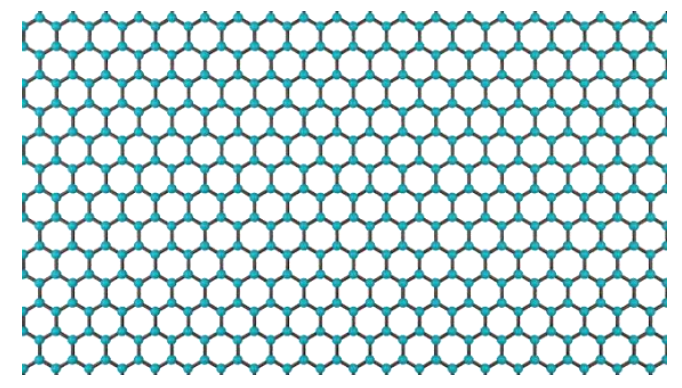

Şekil 1. Grafenin yapısı

$\mathrm{Bu}$ çalışma kapsamında, yalnızca karbon atomlarından oluşan iki boyutlu hekzagonal yapıdaki tek katmanlı grafen plakaların eğilme ve titreşim analizleri yerel olmayan elastisite teorileri ile gerçekleştirilecektir. Grafen plakalar ince plak şeklinde modellenecek olup modellemede Kirchhoff ince plak teorisi kullanılacaktır. Ayrıca, bu yapının elastik bir üzerinde olması durumu Winkler ve Pasternak zemin modelleri kullanılarak dikkate alınacaktır.

\section{Materyal ve Yöntem}

Kirchhoff ince plak teorisi olarak da adlandırılan, eğilmenin lineer, elastik ve küçük deformasyon teorisinin geçerli olduğu plak teorisi olduğu kabul edilmiştir. Bu varsayımlardan birçoğu, kirişlerin basit eğilme teorisine benzemektedir. Bu varsayımlar, üç boyutlu plak probleminin iki boyutlu bir probleme indirgenmesine olanak sağlar (Liew ve Han, 1995). Şekil 2'de Winkler-Pasternak zemine oturan dikdörtgen şeklindeki bir grafen tabakası gösterilmiştir. Burada Winkler zemin yay șeklinde, Pasternak zemin ise plak şeklinde gösterilmiştir.

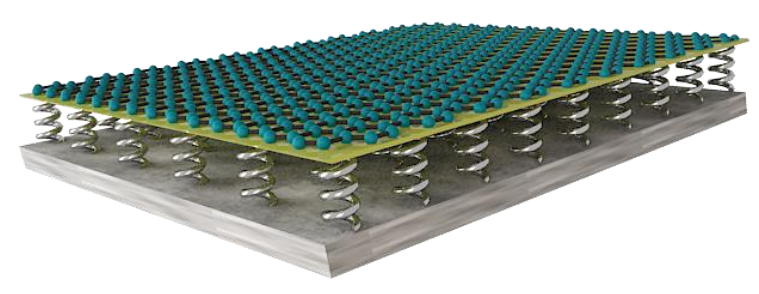

Şekil 2. Winkler-Pasternak zemine oturan dikdörtgen grafen

\subsection{Yerel Olmayan Elastisite Teorisi}

Boyut etkisinin önemli olduğu mikro ve nano ölçekli yapılarda (karbon nanotüp, biyolojik virüslerin ve kanser hücrelerinin matematik modellenmesi, mikrotüpçükler, mikro-elektro aygıtlar, mikro devreler gibi) özellikle Eringen tarafından önerilen yerel olmayan elastisite yaygın olarak kullanılmaktadır (Civalek ve Akgöz, 2010).

Klasik fizik teorilerine göre denge denklemleri maddenin her bir noktası için geçerli olmaktadır. Bu durum madde boyutu büyük olan cisimler için geçerlidir. Fakat boyutlar küçüldükçe maddenin iç yapısını da dikkate alarak referans noktası dışındaki yerlerin de etkileșimini göz önünde bulundurmak yerinde olacaktır. Yerel olmayan elastisite teorisi bir noktadaki gerilmeyi hesap ederken sadece o noktadaki şekil değiştirmelerin bilinmesinin yeterli olmadığına aynı zamanda diğer tüm noktaların şekil değiştirmelerinin bir fonksiyonuna bağlı olması gerektiğine dayanmaktadır. Cisimler yer değiştirdiklerinde cismin şeklinde düzensizlikler meydana gelmektedir. $\mathrm{Bu}$ düzensizlikler de cismin içinde gerilmeler meydana getirmektedir. Buna örnek; bazı malzemelerin şekil değiștirdiğinde iç gerilme ve şekil değiştirme enerjilerinin sonsuza gitmesidir. Yerel olmayan elastisite teorisi ile çözüm yapıldığında bu sorun ortadan kalkar (Tepe, 2007). Özetle atomik yapının bünyesinden meydana gelen gerilmeler ihmal edilebilecek durumdaysa çözüm için klasik elastisite teorisi kullanılırken, gerilmelerin ihmal edilemeyecek kadar büyük olması durumu ve yerel şartların dışındaki şartlarında dikkate alınması gerektiği durumlar için yerel olmayan elastisite teorisi kullanılır. Homojen ve izotrop elastik bir katının yerel olmayan cauchy hareket denklemi (Eringen, 1983) 


$$
\tau_{k l, l}+\rho\left(f_{l}-\frac{\partial^{2} u_{l}}{\partial t^{2}}\right)=0
$$

Bünye denklemi;

$$
\tau_{k l}(x)=\int_{v} \varepsilon_{k l m n}\left(x-x^{l}\right) \varepsilon_{m n} d v\left(x^{l}\right)
$$

şeklindedir. Burada $\tau_{k l}$ gerilme tansörünü, $\rho$ kütle yoğunluğunu, $f_{l}$ kütle kuvveti yoğunluğunu, $u_{l}$ yer değiștirme vektörünü, $v$ elastik cismin kapladı̆̆ hacmi, $t$ zamanı, $\varepsilon_{k l}$ şekil değiştirmeyi ifade etmektedir.

$$
\varepsilon_{k l}=\frac{1}{2}\left(\frac{\partial u_{k}}{\partial x_{l}}+\frac{\partial u_{l}}{\partial x_{k}}\right)
$$

şeklinde olduğu bilinmektedir. $\varepsilon_{\text {klmn }},\left(x-x^{l}\right)$ vektörünün bir fonksiyonu olmak üzere buradan da anlaşılacağı gibi $x$ noktasındaki gerilme $x^{l}$ noktasındaki şekil değiştirmeye de bağlıdır.

$$
\begin{aligned}
& \tau_{k l}\left(x^{l}\right)=\lambda \varepsilon_{m m}\left(\mathrm{x}^{l}\right) \delta_{k l}+2 \mu \varepsilon_{k l}\left(\mathrm{x}^{l}\right) \\
& \varepsilon_{k l}\left(x^{l}\right)=\frac{1}{2}\left(\frac{\partial u_{k}\left(x^{l}\right)}{\partial x_{l}^{l}}+\frac{\partial u_{l}\left(x^{l}\right)}{\partial x_{k}^{l}}\right)
\end{aligned}
$$

Bu denklemlerde $\tau_{k l}\left(x^{l}\right)$ cismin $x^{l}$ noktasındaki klasik (Cauchy) ya da yerel gerilme tansörü, $\varepsilon_{k l}\left(x^{l}\right)$ cismin $x^{l}$ noktasındaki lineer şekil değiștirme tansörü, $\lambda$ ve $\mu$ ise Lame sabitleridir.

Eringen tarafından kurucu denklem aşağıdaki bağıntı ile verilmiştir.

$$
\left[1-\left(e_{0} a\right)^{2} \nabla^{2}\right] \sigma_{k l}=C: \varepsilon
$$

Burada, a değeri malzemenin iç (granüler mesafe, latik parametre C-C karbon moleküleri çapları arasındaki uzaklık) ve dıș karakteristik uzunluğudur (kırılma veya dalga uzunluğu) ve $\mathrm{e}_{0}$ ise her bir malzeme için deneysel olarak belirlenmiş ve önerilmiş olan katsayıdır. C ile ifade edilen ise dördüncü dereceden gerilme tansörüdür. $\quad \nabla$ ise Laplace operatörünü temsil etmektedir ve aşağıdaki şekilde ifade edilebilir.

$$
\nabla^{2}=\frac{\partial^{2}}{\partial x^{2}}+\frac{\partial^{2}}{\partial y^{2}}
$$

İki boyutlu formda yerel olmayan gerilme ifadesi düzenlenirse

$$
\begin{aligned}
\sigma_{x x}-\left(e_{0} a\right)^{2}( & \left.\frac{\partial^{2} \sigma_{x x}}{\partial \mathrm{x}^{2}}+\frac{\partial^{2} \sigma_{x x}}{\partial \mathrm{y}^{2}}\right) \\
& =\frac{E}{1-\vartheta^{2}}\left(\varepsilon_{x x}+\vartheta \varepsilon_{y y}\right)
\end{aligned}
$$

$$
\begin{array}{r}
\sigma_{y y}-\left(e_{0} a\right)^{2}\left(\frac{\partial^{2} \sigma_{y y}}{\partial \mathrm{x}^{2}}+\frac{\partial^{2} \sigma_{y y}}{\partial \mathrm{y}^{2}}\right) \\
=\frac{E}{1-\vartheta^{2}}\left(\vartheta \varepsilon_{x x}+\vartheta \varepsilon_{y y}\right) \\
\tau_{x y}-\left(e_{0} a\right)^{2}\left(\frac{\partial^{2} \tau_{x y}}{\partial \mathrm{x}^{2}}+\frac{\partial^{2} \tau_{x y}}{\partial \mathrm{y}^{2}}\right)=G \gamma_{x y}
\end{array}
$$

\subsection{Formülasyon}

Klasik Kirchoff plak teorisine göre deplasman ifadeleri (Reddy, 1997)

$$
\begin{aligned}
& \mathrm{u}=\mathrm{u}_{0}(\mathrm{x}, \mathrm{y}, \mathrm{t})-\mathrm{z} \frac{\partial w}{\partial x} \\
& \mathrm{v}=\mathrm{v}_{0}(\mathrm{x}, \mathrm{y}, \mathrm{t})-\mathrm{z} \frac{\partial w}{\partial y} \\
& \mathrm{w}=\mathrm{w}(\mathrm{x}, \mathrm{y}, \mathrm{t})
\end{aligned}
$$

$u, v, w$ nanoplağın orta yüzeylerindeki deplasman fonksiyonlarını ifade etmektedir, ' $t$ ' ise zamanı ifade etmektedir. Şekil değiştirmeler ise aşağıdaki şekilde tanımlanabilir

$$
\begin{aligned}
& \varepsilon_{x x}=\frac{\partial u_{0}}{\partial x}-\mathrm{z} \frac{\partial^{2} w}{\partial x^{2}} \\
& \varepsilon_{y y}=\frac{\partial v_{0}}{\partial x}-\mathrm{z} \frac{\partial^{2} w}{\partial y^{2}} \\
& \varepsilon_{z z}=0 \\
& \gamma_{x y}=\left(\frac{\partial u}{\partial y}+\frac{\partial v}{\partial x}\right)-2 \mathrm{z} \frac{\partial^{2} w}{\partial x \partial y} \\
& \gamma_{x z}=0, \gamma_{y z}=0
\end{aligned}
$$

Toplam potansiyel enerji ifadesi en genel haliyle aşağıdaki gibi ifade edilebilir

$$
\Pi=\mathrm{T}-(U+V)
$$

Burada U potansiyel enerjiyi, T kinetik enerjiyi ifade etmektedir. $\mathrm{V}$ ise dıș kuvvetlerin ve elastik ortamın yaptığı işi göstermektedir. Kinetik enerji ifadesi en genel anlamda

$$
\mathrm{T}=\frac{1}{2} \int_{0}^{L} \int_{A} \rho\left(\dot{u}^{2}+\dot{v}^{2}+\dot{w}^{2}\right) d z d A
$$

şeklinde tanımlanır. Şekil değiştirme ifadesi 


$$
\begin{array}{r}
\mathrm{U}=\frac{1}{2} \int_{0}^{L} \int_{A}\left(\sigma_{x x} \varepsilon_{x x}+\sigma_{y y} \varepsilon_{y y}\right. \\
\left.+\tau_{x y} \gamma_{x y}\right) d x d A
\end{array}
$$

ve Pasternak zemin etkisi ve dış yüklerden dolayı oluşan kuvvet

$$
\begin{array}{r}
\mathrm{V}=\frac{1}{2} \int_{0}^{L} \int_{A}\left(-q(x)+k_{w} w\right. \\
\left.-k_{G} \nabla^{2} w\right) d x d A
\end{array}
$$

olarak tanımlanabilir. Burada $k_{w}$ ve $k_{G}$ zemin için Winkler ve Pasternak katsayıları, $q(x)$ yayılı yükü ve $\rho$ ise yoğunluğu ifade etmektedir. Klasik Kirchhoff plak teorisine göre kuvvet ve eğilme momenti ile gerilme arasındaki ilişki aşağıdaki gibi tanımlanabilir

$$
\begin{aligned}
& N_{x x}=\int_{-h / 2}^{h / 2} \sigma_{x x} d z, \quad N_{y y}=\int_{-h / 2}^{h / 2} \sigma_{y y} d z \\
& N_{x y}=\int_{-h / 2}^{h / 2} \tau_{x y} d z \\
& M_{x x}=\int_{-h / 2}^{h / 2} z \sigma_{x x} d z, \quad M_{y y}=\int_{-h / 2}^{h / 2} z \sigma_{y y} d z \\
& M_{x y}=\int_{-h / 2}^{h / 2} z \tau_{x y} d z
\end{aligned}
$$

Denklem (14-16) yardımıyla virtüel iş prensibi üzerinden plak hareket denklemi her üç deplasman doğrultusunda (Reddy, 1997)

$$
\begin{aligned}
& \frac{\partial N_{x x}}{\partial x}+\frac{\partial N_{x y}}{\partial y}=m_{0} \frac{\partial^{2} u_{0}}{\partial t^{2}} \\
& \frac{\partial N_{y y}}{\partial y}+\frac{\partial N_{x y}}{\partial x}=m_{0} \frac{\partial^{2} v_{0}}{\partial t^{2}} \\
& \frac{\partial^{2} M_{x x}}{\partial x^{2}}+2 \frac{\partial^{2} M_{x y}}{\partial x \partial y}+\frac{\partial^{2} M_{y y}}{\partial y^{2}} \\
& +N_{x x} \frac{\partial^{2} w}{\partial x^{2}}+2 N_{x y} \frac{\partial^{2} w}{\partial x \partial y}+N_{y y} \frac{\partial^{2} w}{\partial y^{2}} \\
& +q(x)+k_{w} w-k_{G}\left(\frac{\partial^{2} w}{\partial x^{2}}+\frac{\partial^{2} w}{\partial y^{2}}\right) \\
& =\rho h \frac{\partial^{2} w}{\partial t^{2}}
\end{aligned}
$$

şeklindedir. Denklem (17)'nin denklem (8-10)'da yerine yazılmasıyla yerel olmayan moment ifadeleri

$$
\begin{aligned}
& M_{x x}-\left(e_{0} a\right)^{2}\left(\frac{\partial^{2} M_{x x}}{\partial x^{2}}+\frac{\partial^{2} M_{x x}}{\partial y^{2}}\right)=-D\left(\frac{\partial^{2} w}{\partial x^{2}}+v \frac{\partial^{2} w}{\partial y^{2}}\right) \\
& M_{y y}-\left(e_{0} a\right)^{2}\left(\frac{\partial^{2} M_{y y}}{\partial x^{2}}+\frac{\partial^{2} M_{y y}}{\partial y^{2}}\right)=-D\left(\frac{\partial^{2} w}{\partial y^{2}}+v \frac{\partial^{2} w}{\partial x^{2}}\right)
\end{aligned}
$$

$$
M_{x y}-\left(e_{0} a\right)^{2}\left(\frac{\partial^{2} M_{x y}}{\partial x^{2}}+\frac{\partial^{2} M_{x y}}{\partial y^{2}}\right)=-D(1-v) \frac{\partial^{2} w}{\partial x \partial y}
$$

halini alır. Burada $D=\frac{E h^{3}}{12\left(1-v^{2}\right)}$ 'yi ifade etmektedir. Denklem (19), denklem (18)'deki yerine yazılırsa (kuvvet ifadeleri sıfır alınarak) yönetici denklem (Pradhan ve Kumar, 2011; Pradhan ve Phadikar, 2009a-b; Phadikar ve Pradhan, 2010; Pradhan ve Murmu, 2009a-d; Mohammadimehr vd., 2014)

$$
\begin{aligned}
& D\left(\frac{\partial^{4} w}{\partial x^{4}}+2 \frac{\partial^{4} w}{\partial x^{2} \partial y^{2}}+\frac{\partial^{4} w}{\partial y^{4}}\right) \\
& -\left(e_{0} a\right)^{2}\left[\begin{array}{l}
m_{0}\left(\frac{\partial^{4} w}{\partial x^{2} \partial t^{2}}+\frac{\partial^{4} w}{\partial y^{2} \partial t^{2}}\right)+k_{w}\left(\frac{\partial^{2} w}{\partial x^{2}}+\frac{\partial^{2} w}{\partial y^{2}}\right) \\
-k_{G}\left(\frac{\partial^{4} w}{\partial x^{4}}+2 \frac{\partial^{4} w}{\partial x^{2} \partial y^{2}}+\frac{\partial^{4} w}{\partial y^{4}}\right) \\
-q(x)\left(\frac{\partial^{2} w}{\partial x^{2}}+\frac{\partial^{2} w}{\partial y^{2}}\right)
\end{array}\right] \\
& -q(x)+k_{w} w-k_{G}\left(\frac{\partial^{2} w}{\partial x^{2}}+\frac{\partial^{2} w}{\partial y^{2}}\right)+\rho h \frac{\partial^{2} w}{\partial t^{2}}=0
\end{aligned}
$$

olarak bulunmuş olur.

\subsection{Analitik Çözüm}

Çözüm için dört kenarı basit mesnetli plak (SSSS) düşünülmüştür. Deplasman ve frekansın elde edilmesi için kullanılacak olan Navier tipi çözüm yöntemi aşağıdaki şekilde tanımlanmaktadır.

$$
w=\sum_{m=1}^{\infty} \sum_{n=1}^{\infty} W_{m n} \sin \left(\alpha_{m} x\right) \sin \left(\beta_{n} y\right) e^{i \omega t}
$$

Burada $\alpha_{m}=\frac{m \pi}{a}, \beta_{n}=\frac{n \pi}{b}$ 'dir

Denklem (20)'de q(x) deplasman ifadesi sıfır(0) alınarak Denklem (21), denklem (20)'de yerine yazılırsa

$$
\begin{aligned}
& D\left(\alpha_{m}{ }^{2}+\beta_{n}{ }^{2}\right)^{2} \\
&+ k_{w}\left(1+\left(e_{0} a\right)^{2}\left(\alpha_{m}{ }^{2}+\beta_{n}{ }^{2}\right)\right) \\
& \omega^{2}=+k_{G}\left(\alpha_{m}{ }^{2}+\beta_{n}{ }^{2}\right)\left(1+\left(e_{0} a\right)^{2}\left(\alpha_{m}{ }^{2}+\beta_{n}{ }^{2}\right)\right) \\
& \rho h\left(1+\left(e_{0} a\right)^{2}\left(\alpha_{m}{ }^{2}+\beta_{n}{ }^{2}\right)\right)
\end{aligned}
$$

şeklinde frekans ifadesi elde edilmiş olur. Basit mesnetli grafenin üzerindeki yayılı yükün tanımlaması aşağıdaki şekilde yapılabilir

$$
q=\sum_{m=1}^{\infty} \sum_{n=1}^{\infty} q_{m n} \sin \left(\alpha_{m} x\right) \sin \left(\beta_{n} y\right)
$$


Burada

$$
q_{m n}=\frac{4}{a b} \int_{0}^{a} \int_{0}^{b} q \sin \left(\alpha_{m} x\right) \sin \left(\beta_{n} y\right) d x d y
$$

olarak elde edilir. Statik analiz için denklem (21) ve (23), denklem (29)'de yerine yazılırsa

$$
\begin{aligned}
W_{m n}= & \frac{q_{m n}\left(1+\left(e_{0} a\right)^{2}\left(\alpha_{m}{ }^{2}+\beta_{n}{ }^{2}\right)\right)}{D\left(\alpha_{m}{ }^{2}+\beta_{n}{ }^{2}\right)^{2}+k_{w}\left(1+\left(e_{0} a\right)^{2}\left(\alpha_{m}{ }^{2}+\beta_{n}{ }^{2}\right)\right)} \\
& +k_{G}\left(\alpha_{m}{ }^{2}+\beta_{n}{ }^{2}\right)\left(1+\left(e_{0} a\right)^{2}\left(\alpha_{m}{ }^{2}+\beta_{n}{ }^{2}\right)\right)
\end{aligned}
$$

şeklinde deplasman ifadesi bulunmuş olur.

\subsection{Ayrık tekil konvolüsyon (ATK) yöntemi}

Ayrık tekil konvolüsyon (ATK) yöntemi ilk olarak Wei (1999) tarafından ortaya atılmıştır. Wei'nin de belirttiği gibi (Wei, 1999; Wei, 2001; Wei vd., 2002ab) çeşitli fen bilimleri ve mühendislik problemlerinde görülen tekil konvolüsyonlar (TK), Hilbert, Abel and Radon dönüşümleri gibi, matematik dönüşümlerinin özel bir sınıfını oluşturur. Gerçekten de çoğu pratik uygulamada bu dönüşümlerin kullanılması gerekir. Wei ve arkadașları Ayrık Tekil Konvolüsyon algoritmasını ilk olarak katı ve akışkanlar mekaniği problemlerinin çözümünde uygulandı (Civalek, 2006; Civalek, 2007; Civalek, 2009; Civalek ve Gürses, 2009; Lim vd., 2005; Baltacıoğlu vd., 2011; Demir vd., 2016; Mercan vd., 2016; Mercan ve Civalek, 2016b). Bundan başka, ATK algoritmasının mekanikte uygulanan türevsel eşitliklerin çözümünde esneklik sağlayan özelliklere sahip olduğu ortaya çıkmaktadır. Diğer sayısal yöntemlerde olduğu gibi ayrık tekil konvolüsyon yönteminde de sürekli model kerneller kullanılarak ayrıștırılır. Kernel olarak Shannon kernel, Shannon delta kernel, Dirichlet kernel, kullanılır. Tekil konvolisyon ( Wei, 1999)

$$
F(t)=(T * \eta)(t)=\int_{-\infty}^{\infty} T(t-x) \eta(x) d x
$$

olarak ifade edilebilir, tekil kernel aşağıdaki formda yazılır

$$
T(x)=\delta^{(n)}(x) \quad(\mathrm{n}=0,1,2, \ldots)
$$

Buradaki ayrık kernel of delta tiptir. Yeterli düzgün yaklaşımla, ayrık tekil konvolüsyon yönteminin dikkate alınması son derece etkili olur (Wei, 1999)

$$
F_{\alpha}(t)=\sum_{k} T_{\alpha}\left(t-x_{k}\right) f\left(x_{k}\right)
$$

Yakın geçmişte, bazı yeni kernellerin kullanımı mekanik ve uygulamalı matematik problemlerinin çözümünde önerilmiştir. Shannon kernel :

$$
\delta_{\Delta, \sigma}\left(x-x_{k}\right)=\frac{\sin \left[(\pi / \Delta)\left(x-x_{k}\right)\right]}{(\pi / \Delta)\left(x-x_{k}\right)} \exp \left[-\frac{\left(x-x_{k}\right)^{2}}{2 \sigma^{2}}\right]
$$

şeklinde düzenlenmiştir. Örneğin bir fonksiyon için herhangi bir mertebeden türev

$$
f^{(n)}(x) \approx \sum_{k=-M}^{M} \delta_{\Delta}\left(x-x_{k}\right) f\left(x_{k}\right)
$$

olarak belirtilmiștir ve (n) türevin mertebesini göstermektedir. $(2 \mathrm{M}+1)$ x çevresinde konumlanan ve genellikle toplam değerinden daha küçük toplam hesap genişliğidir.

\section{Problemin Çözümü}

Bu bölümde bütün kenarları basit mesnetli dikdörtgen şekle sahip tek katmanlı grafen plakanın serbest titreşim ve statik analizi yerel olmayan elastisite teorisine dayalı olarak gerçekleștirilmiştir. Yerel olmayan malzeme ve elastik zemin parametrelerinin grafen plakaya ait açısal frekans ve deplasman değerleri üzerindeki etkileri detaylı bir biçimde incelenmiştir. Tablolarda yer alan boyutsuz zemin parametreleri $K_{W}=\frac{k_{W} a^{4}}{D}$ ve $K_{G}=\frac{k_{G} a^{2}}{D}$ şeklinde ifade edilebilir.

Tablo 1. Winkler zemine oturan kare bir grafen tabakanın boyutsuz temel titreşim frekansı

$$
\left(\Omega=\omega a^{2} \sqrt{\frac{\rho h}{D}}\right)
$$

\begin{tabular}{cccc}
\hline $\mathrm{K}_{\mathrm{w}}$ & SSSS & SCSC & SSSC \\
\hline 0 & 19.8073 & 28.3433 & 23.6730 \\
100 & 22.1314 & 30.6412 & 25.6832 \\
1000 & 37.2781 & 42.8571 & 39.5003 \\
\hline
\end{tabular}

Tablo 1'de b/a oranı 1 olan Winkler zemine oturan grafen tabakanın boyutsuz temel frekans değerleri yer almaktadır. Winkler zemin parametresi değerinin artması bütün mesnet koşullarında boyutsuz temel titreşim frekansı değerini artırmaktadır. Winkler zemin etkili veya etkisiz en düşük boyutsuz temel titreşim frekansı değerleri SSSS mesnet koşulunda çıkarken en yüksek değerler SCSC mesnet koşulunda gözlenmiştir. Tablo 2'de SSSS mesnet koşuluna sahip Winkler zemine oturan kare bir grafen tabakanın ilk 5 modu için boyutsuz titreşim frekans değerleri görülmektedir. Winkler zemin etkisi dahilinde mod sayısı değeri arttıkça frekans değerleri artmaktadır. Winkler zemin parametresi değerinin artması da frekans değerlerini artırmakla birlikte yüksek 
modlarda bu parametrenin etkisinin azaldığı gözlenmektedir.

Tablo 2. Winkler elastik zemine oturan SSSS sinır şartına sahip grafen tabakanın boyutsuz frekans değerleri $(b / a=1)$

\begin{tabular}{ccccc}
\hline \multirow{2}{*}{$K_{w}$} & \multicolumn{4}{c}{ mod sayıs } \\
\cline { 2 - 5 } & 1 & 2 & 3 & 4 \\
\hline 100 & 22.1314 & 50.2861 & 79.6771 & 99.8913 \\
500 & 29.8758 & 54.2032 & 82.1412 & 101.6573 \\
\hline
\end{tabular}

Tablo 3. İki parametreli elastik matris yapı üzerindeki grafen tabakanın boyutsuz temel frekans değerleri

\begin{tabular}{ccccc}
\hline \multicolumn{2}{c}{ zemin } & & & \\
\multicolumn{2}{c}{ parametreleri } & SSSS & SCSC & SSSC \\
\cline { 1 - 2 } $\mathrm{K}_{\mathrm{W}}$ & $\mathrm{K}_{\mathrm{G}}$ & & & \\
\hline 0 & 0 & 19.8073 & 28.3433 & 23.6730 \\
0 & 100 & 48.6233 & 54.7412 & 51.3345 \\
100 & 100 & 49.6378 & 55.6521 & 52.3145 \\
\hline
\end{tabular}

Tablo 3'te Winkler ve Pasternak zemin etkisindeki kare bir grafen tabakanın boyutsuz temel frekans değerleri bulunmaktadır. Pasternak ve Winkler zemin parametreleri ayrı ayrı bütün mesnet koșullarında boyutsuz temel titreșim frekans değerlerini artırırken aynı değere sahip olduklarında Pasternak zemin parametresinin frekans değerini artırmada daha baskın olduğu görülmektedir. Tablo 4'e benzer olarak en düşük boyutsuz temel frekans değeri SSSS mesnet koşulunda iken yine en yüksek değerler SCSC mesnet koşuluna sahip bir grafen tabakada görülmektedir.

Tablo 4. İki parametreli elastik zemine oturan SSSS sınır șartına sahip grafen tabakanın boyutsuz deplasman değerleri $\left(\delta=W D / q a^{4}\right)$

\begin{tabular}{lll}
\hline $\mathrm{K}_{\mathrm{W}}$ & $\mathrm{K}_{\mathrm{G}}$ & $\delta$ \\
\hline \multirow{2}{*}{1} & 5 & 0.00346 \\
& 20 & 0.00251 \\
75 & 5 & 0.00281 \\
& 20 & 0.00181 \\
\multirow{2}{*}{500} & 5 & 0.00130 \\
& 20 & 0.00114 \\
\hline
\end{tabular}

Tablo 4'te iki parametreye sahip grafen tabakanın SSSS mesnet koşulunda yaptığı deplasmanlar bulunmaktadır. Winkler zemin parametresi değerinin artması deplasman değerini düşürmektedir. Yine sabit bir Winkler zemin parametresi etkisinde Pasternak zemin parametresi değerinin artması boyutsuz deplasman değerini düşürmektedir. Pasternak zemin parametresinin etkisi, Winkler zemin parametresinin düşük olduğu yerlerde daha fazla belli olmaktadır.
Tablo 5. İki parametreli elastik zemine oturan SSSS sınır şartına sahip grafen tabakanın farklı malzeme katsayısında boyutsuz deplasman değerleri

\begin{tabular}{cccccc}
\hline \multirow{2}{*}{$\mathrm{K}_{\mathrm{W}}$} & $\mathrm{K}_{\mathrm{G}}$ & \multicolumn{4}{c}{$\mu^{2}=\left(e_{0} a\right)^{2}$} \\
\cline { 3 - 6 } & & 0 & 1 & 2 & 4 \\
\hline 0 & 0 & 0.0043 & 0.0118 & 0.0193 & 0.0269 \\
& & 8 & 6 & 4 & \\
10 & 0 & 0.0034 & 0.0068 & 0.0088 & 0.0101 \\
0 & & 4 & 5 & 3 & \\
20 & 0 & 0.0028 & 0.0048 & 0.0057 & 0.0062 \\
0 & & 4 & 1 & 0 & 8 \\
10 & 1 & 0.0024 & 0.0037 & 0.0042 & 0.0045 \\
0 & 0 & 3 & 4 & 6 & 1 \\
20 & 1 & 0.0021 & 0.0030 & 0.0033 & 0.0035 \\
0 & 0 & 2 & 5 & 8 & 7 \\
\hline
\end{tabular}

Tablo 5'de yerel olmayan elastisite teorisine göre iki farklı zemin etkisinde olan SSSS mesnet koşuluna sahip grafen tabakanın farklı malzeme katsayısındaki boyutsuz deplasman değerleri görülmektedir. Yerel olmayan malzeme katsayısı dahilinde veya dahil olmadan Winkler ve Pasternak zemin parametrelerinin artmasıyla boyutsuz deplasman değerleri azalmaktadır. Zemin etkili veya etkisiz yerel olmayan malzeme katsayısı arttıkça deplasman değerleri artmaktadır. Yerel olmayan malzeme katsayısı değeri arttıkça Winkler ve Pasternak zeminin etkisi daha fazla olmakta, deplasmanlar arasındaki fark artmaktadır. Genel olarak bakıldığında düşük Pasternak zemin parametresi daha yüksek olan Winkler zemin parametrelerini baskılamakta deplasmandaki azalma oranını daha düşük seviyelere indirmektedir.

Tablo 6. İki parametreli elastik zemine oturan SSSS sınır şartına sahip grafen tabakanın farklı malzeme katsayısında boyutsuz açısal frekans değerleri $(a=b=20 h, h=0.34 \mathrm{~nm})$

\begin{tabular}{ccllll}
\hline \multirow{2}{*}{$\mathrm{K}_{\mathrm{W}}$} & \multirow{2}{*}{$\mathrm{K}_{\mathrm{G}}$} & \multicolumn{5}{c}{$\mu^{2}=\left(e_{0} a\right)^{2}$} \\
\cline { 3 - 6 } & & \multicolumn{1}{c}{0} & \multicolumn{1}{c}{1} & \multicolumn{1}{c}{4} \\
\hline 0 & 0 & 19.739 & 16.524 & 14.497 & 11.996 \\
& & 2 & 8 & 8 & 2 \\
10 & 0 & 22.127 & 19.315 & 17.612 & 15.617 \\
0 & & 7 & 0 & 1 & 5 \\
20 & 0 & 24.282 & 21.750 & 20.253 & 18.544 \\
0 & & 4 & 1 & 0 & 7 \\
10 & 1 & 26.211 & 23.884 & 22.529 & 21.007 \\
0 & 0 & 2 & 3 & 5 & 1 \\
20 & 1 & 28.054 & 25.893 & 24.649 & 23.265 \\
0 & 0 & 0 & 2 & 1 & 8 \\
\hline
\end{tabular}

Tablo 6'da iki parametreye sahip grafen tabakanın SSSS mesnet koşulunda serbest titreşim açısal frekansları yer almaktadır. Winkler ve Pasternak zemin parametresi değerinin artması frekansları artırmaktadır. Yerel olmayan malzeme katsayısı 
arttıkça ise açısal frekans değerlerinin azaldığı görülmektedir.

\section{Sonuç ve Tartışma}

Bu çalıșmada, elastik zemin üzerindeki tek katmanlı grafen plakaya ait eğilme ve serbest titreşim analizleri gerçekleştirilmiştir. Grafen plak Kirchhoff plak teorisine göre modellenmiştir. Elastik zemin etkisi Winkler-Pasternak elastik zemin modelleri ile hesaba dahil edilmiștir. Yerel olmayan elastisite ile elde edilen sonuçlar klasik sonuçlarla karşılaştırmalı biçimde tablolar ile sunulmuştur. Yapılan analizler sonucu ulaşılan sonuçlar incelendiğinde elastik zemin etkisinin dikkate alınmasıyla birlikte deplasman değerlerinin azaldığı temel frekans değerlerinin ise arttığı gözlemlenmiştir. Göz önüne alınan yapıların karakteristik boyutlarında meydana gelen artış sonucunda boyut etkisi giderek azalmakta olduğu ve elde edilen sonuçların klasik sonuçlara yaklaştığı görülmüştür.

\section{Teşekkür}

Katkılarından dolayı Akdeniz Üniversitesi BAP Birimine teșekkürlerimizi sunarız.

\section{Conflict of Interest / Çıkar Çatışması}

Yazarlar tarafından herhangi bir çıkar çatışması beyan edilmemiştir.

No conflict of interest was declared by the authors.

\section{Kaynaklar}

Aghababaei, R., Reddy, J.N., 2009. Nonlocal third-order shear deformation plate theory with application to bending and vibration of plates. Journal of Sound and Vibration, 326, 277-289.

Akbaș, Ş.D., 2016a. Analytical solutions for static bending of edge cracked micro beams. Structural Engineering and Mechanics. 59(3), 579-599.

Akbaş, Ş.D. 2016b. Free vibration of edge cracked functionally graded microscale beams based on the modified couple stress theory. International Journal of Structural Stability and Dynamics, (doi: 10.1142/S021945541750033X)

Akbaş, Ş.D., 2016c. Static analysis of a nano plate by using generalized differantial quadrature method. International Journal of Engineering and Applied Sciences. 8(2), 30-39.

Akgöz, B., Civalek, Ö., 2012. Free vibration analysis for single-layered graphene sheets in an elastic matrix via modified couple stress theory. Mater. Des., 42, 164-171.
Akgöz, B., Civalek, Ö.,2014. Shear deformation beam models for functionally graded microbeams with new shear correction factors, Composite Structures, 112, 214-225

Aksencer, T., Aydoğdu, M., 2011. Levy type solution method for vibration and buckling of nanoplates using nonlocal elasticity theory. Physica E, 43, 954959.

Alibeigloo, A., 2011. Free vibration analysis of nanoplate using three-dimensional theory of elasticity. Acta Mechanica, 222, 149-159.

Ansari, R., Arash, B., Rouhi, H., 2011. Vibration characteristics of embedded multi-layered graphene sheets with different boundary conditions via nonlocal elasticity. Composite Structures, 93, 2419-2429.

Ansari, R., Rajabiehfard, B., Arash, B., 2010a. Nonlocal finite element model for vibrations of embedded multi-layered graphene sheets. Computational Materials Science, 49, 831-838.

Ansari, R., Sahmani, S., Arash, B., 2010b. Nonlocal plate model for free vibrations of single-layered graphene sheets. Physics Letters A, 375, 53-62.

Ansari, R., Rouhi, H., 2012. Explicit analytical expressions for the critical buckling stresses in a monolayer graphene sheet based on nonlocal elasticity. Solid State Communications, 152, 56-59.

Avcar, M., 2010. Elastik zemin üzerinde bulunan her iki ucu ankastre mesnetli rastgele ve sürekli homojen olmayan kirişin titreşim analizi. Süleyman Demirel Üniversitesi Mühendislik Bilimleri ve Tasarım Dergisi, 1(1), 33-38.

Avcar, M., 2016a. Effects of material non-homogeneity and two parameter elastic foundation on fundamental frequency parameters of timoshenko beams. Acta Physica Polonica A, 130(1), 375-378

Avcar, M., 2016b. Pasternak zemine oturan eksenel yüke maruz homojen olmayan kirişin serbest titreșimi. Politeknik Dergisi, 19(4) .

Babaei, H., Shahidi, A.R., 2011. Small-scale effects on the buckling of quadrilateral nanoplates based on nonlocal elasticity theory using the Galerkin method. Arch. Appl. Mech., 81, 1051-1062.

Baltacıoğlu, A.K., Civalek, Ö., Akgöz, B., Demir, F., 2011. Large deflection analysis of laminated composite plates resting on nonlinear elastic foundations by the method of discrete singular convolution. International Journal of Pressure Vessels and Piping, 88, 8, 290-300.

Civalek, Ö., 2006. The determination of frequencies of laminated conical shells via the discrete singular convolution method. Journal of Mechanics of Materials and Structures, 1(1), 163-182.

Civalek, Ö., 2007., Free vibration and buckling analysis of composite plates with straight-sided 
quadrilateral domain based on DSC approach. Finite Elements in Analysis and Design, 43(13), 1013-1022.

Civalek, Ö., 2009. Fundamental frequency of isotropic and orthotropic rectangular plates with linearly varying thickness by discrete singular convolution method. Applied Mathematical Modelling, 33(10), 3825-3835.

Civalek, Ö., Akgöz, B., 2010. Mechanical modeling of microtubules based on nonlocal continuum theory. In 2010 15th National Biomedical Engineering Meeting.

Civalek, Ö., Akgöz, B., 2013. Vibration analysis of micro-scaled sector shaped graphene surrounded by an elastic matrix, Computational Materials Science, 77, 295-303.

Civalek, Ö., Demir, Ç., 2009. Elastik zemine oturan kiriĢlerin ayrık tekil konvolüsyon ve harmonik diferansiyel quadrature yöntemleriyle analizi. BAÜ FBE Dergisi, 11(1), 56-71.

Civalek, Ö., Demir, Ç., 2011. Buckling and bending analyses of cantilever carbon nanotubes using the Euler-Bernoulli beam theory based on non-local continuum model. Asian Journal of Civil Engineering, 12(5), 651-661.

Civalek, Ö., Demir, Ç., 2016. A simple mathematical model of microtubules surrounded by an elastic matrix by nonlocal finite element method. Applied Mathematics and Computation, 289, 335-352.

Civalek, Ö., Gürses, M., 2009. Free vibration analysis of rotating cylindrical shells using discrete singular convolution technique. International Journal of Pressure Vessels and Piping, 86(10), 677-683.

Demir, Ç., Civalek, Ö. 2015. Nonlocal deflection of microtubules under point load. International Journal of Engineering \& Applied Sciences, 7(1), 33-39.

Demir, Ç., Civalek, Ö., 2016. Nonlocal finite element formulation for vibration. International Journal of Engineering \& Applied Sciences (IJEAS), 8 (2), 109117.

Demir, Ç., Mercan, K., Civalek, Ö., 2016. Determination of critical buckling loads of isotropic, FGM and laminated truncated conical panel. Composites Part B: Engineering, 94, 1-10.

Eringen, A. C. 1983. On differential equations of nonlocal elasticity and solutions of screw dislocation and surface waves. Journal of applied physics, 54(9), 4703-4710.

Eringen, A. C., Edelen, D. G. B., 1972. On nonlocal elasticity. International Journal of Engineering Science, 10(3), 233-248.

Farajpour, A., Danesh, M., Mohammadi, M., 2011a. Buckling analysis of variable thickness nanoplates using nonlocal continuum mechanics. Physica E, 44, 719-727.

Farajpour, A., Shahidi, A.R., Mohammadi, M., Mahzoon, M., 2011b. Axisymmetric buckling of the circular graphene sheets with the nonlocal continuum plate model. Physica E, 43, 1820-1825.

Hashemi, S.H., Samaei, A.T., 2011. Buckling analysis of micro/nanoscale plates via nonlocal elasticity theory. Physica E, 43, 1400-1404.

Jomehzadeh, E., Noori, H.R., Saidi, A.R., 2011. The sizedependent vibration analysis of micro-plates based on a modified couple stress theory. Physica E, 43, 877-883.

Kocatürk, T., Akbaş, Ş.D., 2013. Wave propagation in a microbeam based on the modified couple stress theory. Structural Engineering and Mechanics, 46(3), 417-431

Liang, Y. C., Dou, J. H., Bai, Q. S., 2007. Molecular dynamic simulation study of AFM single-wall carbon nanotube tip-surface interactions. In Key Engineering Materials Trans Tech Publications, 339, 206-210.

Liew, K. M., Han, J.B., 1995. Bending analysis of simply supported shear deformable skew plates. Journal of Engineering Mechanics ASCE, 123, 214-221.

Liew, K.M., Hu, Y.G., He, X.Q., 2008. Flexural wave propagation in single-walled carbon nanotubes, Journal of Computational and Theoretical Nanoscience, 5, 581-586.

Liew, K.M., Hung, K.C., Lim, M.K., 1995. Vibration characteristic of simply supported thick skew plates in three-dimensional setting. Journal of Applied Mechanics ASME, 62, 880-886.

Liew, K.M., Wang, Q., 2007a. Application of nonlocal continuum mechanics to static analysis of microand nano-structures. Physics Letters A, 363, 236242 .

Liew, K.M., Wang, Q., 2007b. Analysis of wave propagation in carbon nanotubes via elastic shell theories. International Journal of Engineering Science, 45, 227-241.

Lim, C.W., Li, Z.R., Wei, GW., 2005. DSC-Ritz method for high-mode frequency analysis of thick shallow shells. International Journal for Numerical Methods in Engineering, 62, 205-232.

Lim, C.W., Liew, K.M., 1995. Vibration of pretwisted cantilever trapezoidal symmetric laminates. Acta Mechanica, 111, 193-208.

Lim, C.W., Liew, K.M., Kitipornchai, S., 1996. Vibration of arbitrarily laminated plates of general trapezoidal planform. Journal of the Acoustical Society of America, 100, 3674-3685.

Ma, H.M., Gao, X.-L., Reddy, J.N., 2008. A microstructure-dependent Timoshenko beam 
model based on a modified couple stress theory. J. Mech. Phys. Solids, 56 , 3379-3391.

Malekzadeh, P., Setoodeh, A.R. Beni A.A., 2011a. Small scale effect on the thermal buckling of orthotropic arbitrary straigh-sided quadrilateral nanoplates embedded in an elastic medium. Composite Structures, 93, 2083-2089.

Malekzadeh, P., Setoodeh, A.R. Beni, A.A., 2011b. Small scale effect on the free vibration of orthotropic arbitrary straight-sided quadrilateral nanoplates. Composite Structures, 93, 1631-1639.

Mercan, K., Civalek, Ö., 2016a. Buckling Analysis of Silicon Carbide Nanotubes (SiCNTs). International Journal of Engineering \& Applied Sciences (IJEAS), 8 (2), 101-108.

Mercan, K., Civalek, Ö., 2016b. DSC method for buckling analysis of boron nitride nanotube (BNNT) surrounded by an elastic matrix. Composite Structures, 143, 300-309.

Mercan, K., Demir, Ç., Civalek, Ö., 2016. Vibration analysis of FG cylindrical shells with power-law index using discrete singular convolution technique. Curved and Layered Structures, 3(1).

Mohammadimehr, M., Najafabadi, M.M.M., Nasiri, H., Navi, B. R., 2014. Surface stress effects on the free vibration and bending analysis of the nonlocal single-layer graphene sheet embedded in an elastic medium using energy method. Proceedings of the Institution of Mechanical Engineers, Part N: Journal of Nanoengineering and Nanosystems, 1740349914559042.

Narendar, S., 2011. Buckling analysis of micro/nanoscale plates based on two-variable refined plate theory incorporating nonlocal scale effects. Composite Structures, 93, 3093-3103.

Phadikar, J.K., Pradhan, S.C., 2010. Variational formulation and finite element analysis for nonlocal elastic nanobeams and nanoplates. Computational Materials Science, 49, 492-499

Pradhan, S.C., Kumar, A., 2010. Vibration analysis of orthotropic graphene sheets embedded in Pasternak elastic medium using nonlocal elasticity theory and differential quadrature method. Computational Materials Science, 50, 239-245.

Pradhan, S.C., Kumar, A., 2011. Vibration analysis of orthotropic graphene sheets using nonlocal elasticity theory and differential quadrature method. Composite Structures, 93, 774-779

Pradhan, S.C., Murmu, T., 2009a. Buckling of biaxially compressed orthotropic plates at small scales. Mechanic Research Communications, 36, 933-938.

Pradhan, S.C., Murmu, T., 2009b. Small scale effect on free in-plane vibration of nanoplates by nonlocal continuum model. Physica E, 41, 1628-1633.
Pradhan, S.C., Murmu, T., 2009c. Small scale effect on the buckling of single-layered graphene sheets under biaxial compression via nonlocal continuum mechanics. Computational Materials Science, 47, 268-274

Pradhan, S.C., Murmu, T., 2009d. Vibration analysis of nanoplates under uniaxial prestressed conditions via nonlocal elasticity. Journal of Applied Physics, 106, 104301.

Pradhan, S. C., Murmu, T., 2010. Small scale effect on the buckling analysis of single-layered graphene sheet embedded in an elastic medium based on nonlocal plate theory. Physica E: Low-dimensional Systems and Nanostructures, 42(5), 1293-1301.

Pradhan, S.C., Phadikar, J.K., 2009a. Nonlocal elasticity theory for vibration of nanoplates. Journal of Sound and Vibration, 325, 206-223.

Pradhan, S.C., Phadikar, J.K., 2009b. Small scale effect on vibration of embedded multilayered graphene sheets based on nonlocal continuum models. Phys. Lett. A, 373, 1062-1069.

Reddy, J.N., 2007. Nonlocal theories for bending, buckling and vibration of beams. International Journal of Engineering Sciences, 45, 288-307.

Reddy, J.N., Pang, S.D., 2008. Nonlocal continuum theories of beams for the analysis of carbon nanotubes. Journal of Applied Physics, 103, 023511.

Reddy., J.N., 1997. Mechanics of Laminated Composite Plates, Theory and Analysis, Chemical Rubber Company, Boca Raton, FL

Salvetat, J.-P., Bonard, J.-M., Thomson, N.H., Kulik, A.J., Forró, L., Benoit, W., Zuppiroli, L., 1999. Mechanical properties of carbon nanotubes. Applied Physics A, $69,255-260$

Samaei, A.T., Abbasion, S., Mirsayar, M.M., 2011. Buckling analysis of a single-layer graphene sheet embedded in an elastic medium based on nonlocal Mindlin plate theory. Mech. Res. Commun., 38 , 481-485.

Sun, C. T., Zhang, H., 2003. Size-dependent elastic moduli of platelike nanomaterials. Journal of Applied Physics, 93(2), 1212-1218.

Şimșek, M., 2010. Vibration analysis of a single-walled carbon nanotube under action of a moving harmonic load based on nonlocal elasticity theory. Physica E: Low-dimensional Systems and Nanostructures, 43, 182-191.

Tepe, A., 2007. Nanoteknolojide nano ölçekteki yapıların lokal olmayan elastisite çerçevesinde incelenmesi. İstanbul Teknik Üniversitesi, Fen Bilimleri Enstitüsü, Doktora Tezi, 114 s, İstanbul.

Tsiatas, G.C., 2009. A new Kirchhoff plate model based on a modified couple stress theory. Int. J. Solids Struct., 46, 2757-2764. 
Ventsel, E., Krauthammer, T., 2001. Thin plates and shells: Theory, analysis, and applications. Marcel Dekker Inc., USA.

Wang, C.M., Reddy, J.N., Lee, K.H., 2000. Shear deformable beams and plates-Relationship with classical solutions, Elsevier.

Wang, K.F., Wang, B.L., 2011. Vibration of nanoscale plates with surface energy via nonlocal elasticity. Physica E, 44, 448-453.

Wang, Q., Arash, B., 2012. A review on the application of nonlocal elastic models in modeling of carbon nanotubes and graphenes. Elsevier Computational Materials Science, 51, 303-313.

Wang, Y.Z., Li, F.M., Kishimoto K., 2011. Thermal effects on vibration properties of double-layered nanoplates at small scales. Elsevier Composites: Part B, 42, 1311-1317.

Wei, G.W., 1999. Discrete singular convolution for the solution of the Fokker-Planck equations. Journal of Chemical Physics, 110, 8930-8942.

Wei, G.W., Zhao, Y.B., Xiang, Y., 2002a. A novel approach for the analysis of high-frequency vibrations. Journal of Sound and Vibration, 257(2), 207-246.

Wei, G.W., Zhao, Y.B., Xiang, Y., 2002b. Discrete singular convolution and its application to the analysis of plates with internal supports; part 1: theory and algorithm. International Journal for Numerical Methods in Engineering, 55, 913-946.

Wei, GW., 2001. Vibration analysis by discrete singular convolution. Journal of Sound and Vibration, 244, 535-553.

Williams, L., Adams, W. 2007. Nanotechnology Demystified. The McGraw-Hill Companies, USA.

Zhang, Y.Q., Liu, G.R. and Xie, X.Y., 2005. Free transverse vibrations of double-walled carbon nanotubes using a theory of nonlocal elasticity. Physical Review B, 71, 195404.

Zhang, Y.Q., Liu, G.R.,Liu, X., 2007. Thermal effect on transverse vibrations of double-walled carbon nanotubes. Nanotechnology, 18, 445701.

Zhu, R., Pan, E., Roy, A. K., 2007. Molecular dynamics study of the stress-strain behavior of carbonnanotube reinforced Epon 862 composites. Materials Science and Engineering: A, 447(1), 5157. 